\title{
A Bayesian joint probability post-processor for reducing errors and quantifying uncertainty in monthly streamflow predictions
}

\author{
P. Pokhrel, D. E. Robertson, and Q. J. Wang \\ CSIRO Land and Water, Graham Road, Highett, Victoria, Australia \\ Correspondence to: P. Pokhrel (prafullapok@gmail.com) \\ Received: 7 September 2012 - Published in Hydrol. Earth Syst. Sci. Discuss.: 2 October 2012 \\ Revised: 9 January 2013 - Accepted: 25 January 2013 - Published: 22 February 2013
}

\begin{abstract}
Hydrologic model predictions are often biased and subject to heteroscedastic errors originating from various sources including data, model structure and parameter calibration. Statistical post-processors are applied to reduce such errors and quantify uncertainty in the predictions. In this study, we investigate the use of a statistical post-processor based on the Bayesian joint probability (BJP) modelling approach to reduce errors and quantify uncertainty in streamflow predictions generated from a monthly water balance model. The BJP post-processor reduces errors through elimination of systematic bias and through transient errors updating. It uses a parametric transformation to normalize data and stabilize variance and allows for parameter uncertainty in the post-processor. We apply the BJP post-processor to 18 catchments located in eastern Australia and demonstrate its effectiveness in reducing prediction errors and quantifying prediction uncertainty.
\end{abstract}

\section{Introduction}

Streamflow predictions from a hydrological model can be used for wide range of applications including flood forecasting at short time scales to long-term assessments of water resources. Model predictions are subject to errors originating from various sources including input data, model structure and parameters. The model is usually calibrated prior to its application to compensate for these errors, thus reducing uncertainty in the predictions. However, a model being a simplified representation of a system will always contain uncertainty in its predictions (Gupta et al., 2005). Post-processors are statistical models that are applied to model predictions to further reduce errors and to quantify uncertainty in the streamflow predictions (Seo et al., 2006).

Post-processors can reduce errors through elimination of systematic bias and/or by reduction of "short memory" or transient errors (Pagano et al., 2011). The former is generally achieved by using simple statistical approaches like quantile mapping or regression (Hashino et al., 2007; Shi et al., 2008), while the latter is generally achieved by prediction updating (Lekkas et al., 2001; Moraweitz et al., 2011). The prediction updating techniques exploit persistence of residuals to correct for errors using linear or non-linear auto-regressive models (WMO, 1992; Shamseldin and O'Connor, 2001; Xiong and O'Connor, 2002; Pagano et al., 2011). Streamflow predictions, even after bias correction and prediction updating, contain errors that cannot be eliminated, and information on prediction uncertainty is useful for decision makers who use the predictions. Post-processors are generally designed to provide an estimate of the total "lumped" uncertainty in the predictions by constructing statistical models of errors based on model predictions and historical observations (e.g. Krzysztofowicz, 1999; Engeland et al., 2005; Montanari and Grossi, 2008).

In hydrology, post-processors have been mostly used for short-term streamflow or river height forecasting. The examples include Bayesian Forecasting System (BFS; Krzysztofowicz, 1999, 2002; Reggiani and Weerts, 2008), the US National Weather Service (NWS) post-processor (Seo et al., 2006), the General Linear Model Post-Processor (Zhao et al., 2011), the meta-Gaussian post-processor (Montanari and Grossi, 2008) and others. They range in complexity from the NWS post-processor that adopts a fairly simple autoregressive error structure (Seo et al., 2006) to BFS that uses a complex parameterization scheme based on meta-Gaussian 
distributions. Some are primarily intended for uncertainty quantification but also include components for error reduction (e.g. Krzysztofowicz, 1999, 2002).

Methods for parameterization, parameter estimation and calculation of predictive distributions differ among postprocessors, although some common features can be found. Most post-processors produce probabilistic predictive distributions of streamflow (or river height) conditioned on model predictions and recent streamflow observations. They generally assume linear dependence among the variates in a transformed normal space, and most use normal quantile transformation (NQT; Krzysztofowicz, 1997, 1999; Todini, 2008; $\mathrm{Li}$ et al., 2010) to normalize the variables. All assume the estimated values of the parameters (of the post-processors) to be "true" and ignore the uncertainty in estimating their values (Krzysztofowicz, 1999, 2002). For a complex postprocessor like BFS, this (parametric uncertainty) can be substantial (Seo et al., 2006). More importantly, they are all designed to post-process streamflow predictions at daily or subdaily time scales.

For many hydrological applications, such as seasonal streamflow forecasting, water resources and climate change assessments, monthly streamflow volumes are of primary interest. While daily predictions from daily models may be post-processed at the daily time scale and then aggregated to monthly, there is no guarantee that the monthly volumes so produced have reliable uncertainty distributions and the least errors achievable. It is likely much more effective to apply post-processing directly at the monthly time scale, where pre-processed monthly volumes may come either from aggregating daily model outputs or simply from monthly models.

In this study, we investigate the use of a Bayesian joint probability (BJP) modelling approach to post-process model predictions of monthly streamflow volumes. The BJP method was originally developed for forecasting seasonal streamflows in Australia (Wang et al., 2009). Here we apply it for bias correction, prediction updating and uncertainty quantification of monthly streamflow volumes generated from a monthly water balance model. The BJP method uses a parametric transformation to normalize data and stabilize variance. It allows for parameter uncertainty in the postprocessor, and this can be important when dealing with monthly variables, which have far fewer data points than daily variables. In this study, we assess three formulations of the BJP post-processor in their ability to reduce error and quantify uncertainty.

The paper is structured as follows. Section 2 describes the catchments and data used in the study. Section 3 presents the hydrological model used and the formulations of the BJP post-processor. Evaluation of the post-processor is given in Sect. 4 and followed by discussions in Sect. 5. Conclusions are drawn in Sect. 6.

\section{Study area and data}

We test BJP post-processor in 18 catchments located in Queensland, Victoria (including one at the border with New South Wales) and Tasmania (see Fig. 1). The Victorian catchments are further divided into 3 regions: upper Murray, central Victoria and southern Victoria (see Table 1).

The catchments range in size from 127 to $36000 \mathrm{~km}^{2}$. The Queensland catchments are the largest in size and experience a semi-arid type of climate, characterized by low rainfall and high evapotranspiration. The mean annual rainfall is less than $600 \mathrm{~mm}$, and the catchments are dry during the austral winter. In contrast, the Victorian catchments experience a temperate climate, with higher rainfall $(617-1400 \mathrm{~mm})$ occurring during the austral winter and spring. The Tasmanian catchments experience temperate oceanic climate and are the wettest with mean annual rainfall in excess of $1900 \mathrm{~mm}$. The Tasmanian catchments are wet throughout the year.

We use observed monthly streamflow data obtained from various water resource management agencies and the $\mathrm{Bu}$ reau of Meteorology, Australia. For most catchments, with the exception of some in Queensland and Victoria, the data are available from 1950 to 2008 (see Table 1). The monthly catchment average rainfall and potential evapotranspiration for each catchment are calculated from a 5-km gridded dataset available from the Australian Water Availability Project (AWAP; Jones et al., 2009).

\section{Methods}

In each catchment, we calibrate parameters of a hydrologic water balance model and generate streamflow predictions. In the context of this study, we define prediction as one time step ahead of forecast of streamflow, under perfect rainfall forecast. The "raw" deterministic streamflow predictions generated by the model contain errors that are unreconciled during calibration process. The BJP post-processor aims to reduce such errors and quantify uncertainty. This section describes the process of generating streamflow predictions, using a hydrologic model, and their subsequent post-processing.

\subsection{Generation of streamflow predictions using a hydrological model}

We use a monthly model known as WAPABA (WAter PArtition and BAlance; Wang et al., 2011) to generate streamflow predictions. WAPABA is a modified version of the Budyko framework model (Zhang et al., 2008) and consists of two storages and five parameters. The model uses consumption curves to partition water into different components based on the availability of water (supply) and demand. WAPABA has been tested in 331 catchments in Australia and demonstrated to perform well (Wang et al., 2011). 
Table 1. Brief attributes of the 18 catchments used for the study.

\begin{tabular}{llrrrrr}
\hline Catchment & Region & $\begin{array}{c}\text { Available } \\
\text { Record }\end{array}$ & $\begin{array}{r}\text { Catchment } \\
\text { area }\left(\mathrm{km}^{2}\right)\end{array}$ & $\begin{array}{r}\text { Mean annual } \\
\text { rainfall }(\mathrm{mm})\end{array}$ & $\begin{array}{r}\text { Mean annual } \\
\text { flow (mm) }\end{array}$ & $\begin{array}{c}\text { Annual } \\
\text { runoff coeff. }\end{array}$ \\
\hline Burdekin River & Queensland & $1967-2008$ & 36260 & 567 & $76(2765 \mathrm{GL})$ & 0.13 \\
Cape River & Queensland & $1967-2008$ & 16074 & 456 & $23(372 \mathrm{GL})$ & 0.05 \\
Lake Hume & upper Murray & $1950-2008$ & 12184 & 819 & $227(2764 \mathrm{GL})$ & 0.28 \\
Dartmouth Reservoir & upper Murray & $1950-2008$ & 3193 & 1042 & $279(890 \mathrm{GL})$ & 0.27 \\
Kiewa River & upper Murray & $1965-2008$ & 1748 & 1099 & $248(433 \mathrm{GL})$ & 0.23 \\
Ovens River & upper Murray & $1959-2008$ & 7543 & 963 & $175(1320 \mathrm{GL})$ & 0.18 \\
Lake Nillahcootie & central Victoria & $1950-2008$ & 422 & 942 & $150(63 \mathrm{GL})$ & 0.16 \\
Lake Eildon & central Victoria & $1950-2008$ & 3877 & 1104 & $373(1447 \mathrm{GL})$ & 0.34 \\
Goulburn Weir & central Victoria & $1950-2008$ & 7166 & 769 & $188(1349 \mathrm{GL})$ & 0.24 \\
Eppalock Reservoir & central Victoria & $1950-2008$ & 1749 & 630 & $98(172 \mathrm{GL})$ & 0.16 \\
Cairn Curran Reservoir & central Victoria & $1950-2008$ & 1603 & 617 & $72(115 \mathrm{GL})$ & 0.12 \\
Tullaroop Reservoir & central Victoria & $1950-2008$ & 702 & 633 & $77(54 \mathrm{GL})$ & 0.12 \\
Thompson Reservoir & southern Victoria & $1950-2008$ & 487 & 1299 & $485(236 \mathrm{GL})$ & 0.37 \\
Upper Yarra Reservoir & southern Victoria & $1950-2008$ & 336 & 1387 & $443(149 \mathrm{GL})$ & 0.32 \\
Maroondah Reservoir & southern Victoria & $1950-2008$ & 129 & 1351 & $577(74 \mathrm{GL})$ & 0.43 \\
O’Shannassy Reservoir & southern Victoria & $1950-2008$ & 127 & 1404 & $766(97 \mathrm{GL})$ & 0.55 \\
Mersey-Forth HES & Tasmania & $1950-2008$ & 2698 & 1900 & $793(2141 \mathrm{GL})$ & 0.42 \\
King HES & Tasmania & $1950-2008$ & 731 & 2703 & $1724(1260 \mathrm{GL})$ & 0.64 \\
\hline
\end{tabular}

Note: HES stands for Hydro Electric Scheme.

We calibrate WAPABA using the shuffled complex evolution search method (SCE; Duan et al., 1994) for a period of five years. Prior to every model run we allow a fiveyear warm-up period to reduce model sensitivity to state initialization errors. We maximize a scalarized multi-objective measure consisting of a uniformly weighted average of the Nash-Sutcliffe efficiency (NS) coefficient (Nash and Sutcliffe, 1970), the NS of log transformed flows, the Pearson correlation coefficient and a symmetric measure of bias. The NS is an "observed-variance-normalized mean squared error" measure that emphasizes large errors, often occurring during large events. The NS of log-transformed flow emphasizes errors occurring during low flow events. The Pearson correlation measures the co-variability of the simulated and the observed. The symmetrical measure of bias evaluates the match between average simulation and average observation (Wang et al., 2011). We then use the calibrated parameters to produce raw WAPABA streamflow predictions using the observed rainfall.

\subsection{Statistical post-processing}

The BJP modelling approach assumes that a set of predictands, $y(2)$, and their predictors, $y(1)$, follow a joint multivariate normal distribution in a transformed space. Normalization of the variables is achieved by using the log-sinh transformation (Wang et al., 2012). The log-sinh transformation replaces the previously used Yeo-Johnson transformation (Yeo and Johnson, 2000; Wang et al., 2009; Wang and Robertson, 2011). Although both have data normalization and variance stabilization properties, the log-sinh has been shown to outperform the Box-Cox-based Yeo-Johnson transformation when applied to catchments with highly skewed data (Wang et al., 2011). The posterior distribution of the parameters $p\left(\boldsymbol{\theta} \mid Y_{\mathrm{OBS}}\right)$, including mean, variance and transformation parameters for each variable and a correlation matrix for the multivariate normal distribution, is estimated using a Bayesian inference (Eq. 1):

$$
p\left(\boldsymbol{\theta} \mid Y_{\mathrm{OBS}}\right) \propto p(\boldsymbol{\theta}) \cdot p\left(Y_{\mathrm{OBS}} \mid \boldsymbol{\theta}\right)
$$

where $Y_{\mathrm{OBS}}$ contains the historical data of both predictor $y(1)$ and predictand $y(2)$ variables used for model inference, and $\boldsymbol{\theta}$ is the parameter vector. $p(\boldsymbol{\theta})$ is the prior distribution of the parameters of the multivariate normal distribution, representing any information available before the use of historical data, $Y_{\mathrm{OBS}}$. The term $p\left(Y_{\mathrm{OBS}} \mid \boldsymbol{\theta}\right)$ is the likelihood function defining the probability of observing the historical data given the model and the parameter sets. The posterior parameter distribution is approximated by 1000 sets of parameters sampled using a Markov Chain Monte Carlo (MCMC) method.

The posterior predictive density for a new event is given by

$$
\begin{aligned}
& f(y(2) \mid y(1))=p\left(y(2) \mid y(1) ; Y_{\mathrm{OBS}}\right) \\
& \quad=\int p(y(2) \mid y(1), \boldsymbol{\theta}) \cdot p\left(\boldsymbol{\theta} \mid Y_{\mathrm{OBS}}\right) \cdot \mathrm{d} \boldsymbol{\theta} .
\end{aligned}
$$

Details of the method for the numerical evaluation of Eqs. (1) and (2) and the prior distribution of the parameters can be found in Wang et al. (2009) and Wang and Robertson (2011).

To apply BJP as a post-processing tool we implement three methodologies with different combinations of the predictors. 

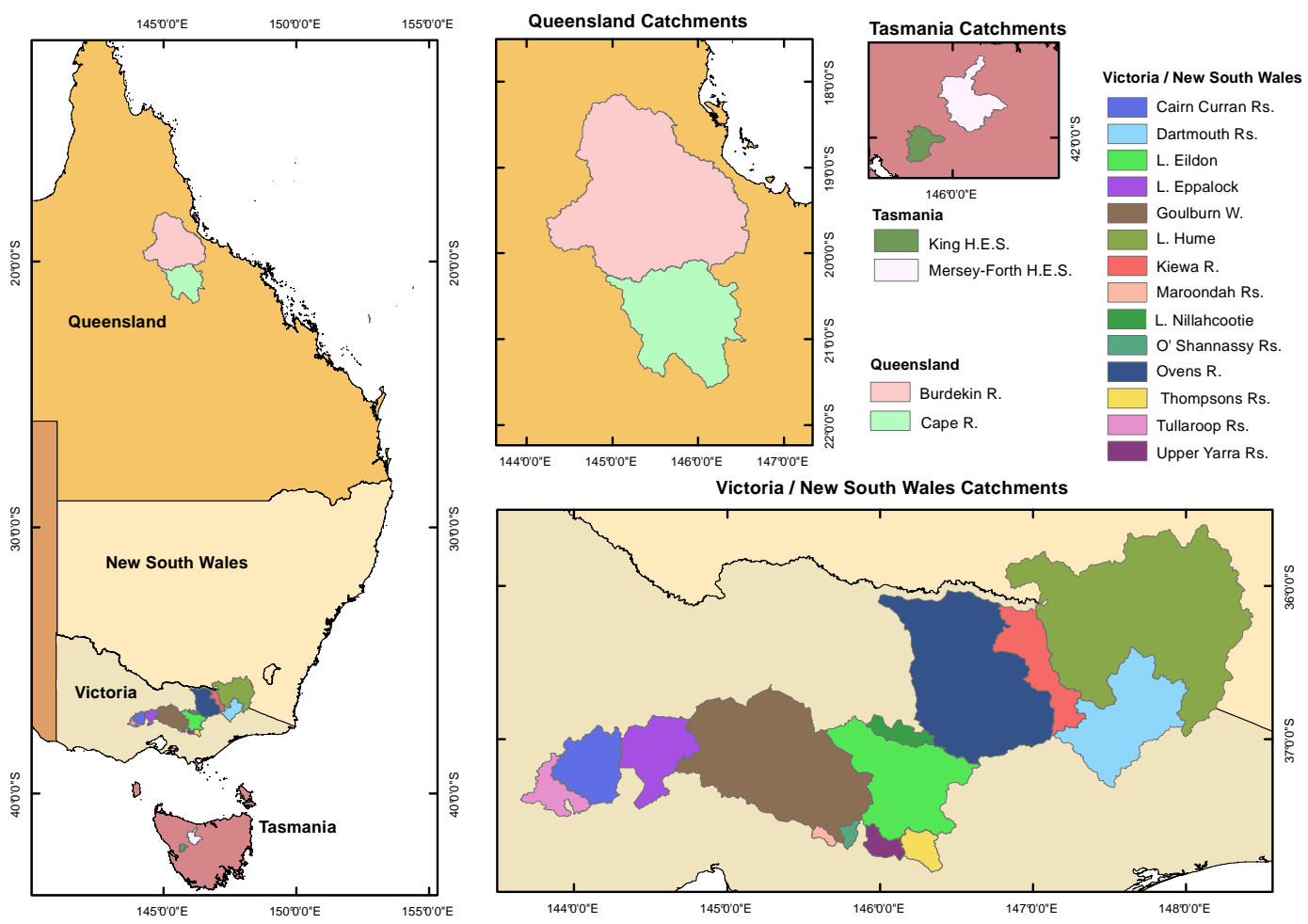

Fig. 1. Location of the 18 catchments used for the study.

\subsubsection{Method A}

Method A represents the simplest case where only WAPABA predictions are applied as the predictor ( $y(1)$ in Eq. 2) and the observed streamflows as the predictand ( $y(2)$ in Eq. 2). This combination is to achieve two post-processing objectives, correction of systematic bias and quantification of uncertainty. The bias correction is achieved through the regression property embedded within the BJP modelling approach (see Wang et al., 2009).

\subsubsection{Method B}

For method B, we add a second predictor over that used for method A. We add streamflow data observed one month previously. The inclusion of lagged streamflow observations is to add an auto-regressive component to the post-processor and allow prediction updating. This method reduces errors through prediction updating as well as correction of systematic bias and quantifies uncertainty.

\subsubsection{Method C}

For method $\mathrm{C}$, we introduce a third predictor, the WAPABA model outputs simulated in the previous month. This inclusion is to further improve the prediction updating ability of the post-processor by utilizing the persistence in the simulated time series.
For each method, we first train the post-processor using the historically observed data. To account for seasonal effects, we establish 12 different models for different months of the year. For each month, the post-processed probabilistic predictions are generated using a "leave-one-out" cross validation procedure. This consists of sampling the parameters using all but the year of interest and then generating predictions for the "left-out" year. The cross validation period in most catchments is about $59 \mathrm{yr}$ (1950-2008).

Figure 2 is an example of the post-processed predictions generated by the BJP post-processor. This example is to provide the reader with an appreciation of how the postprocessed predictions from the BJP post-processor may look. A detailed evaluation of the post-processor, with respect to the post-processing qualities, will be presented in Sect. 4. The example is drawn from Lake Eildon in central Victoria and shows [0.1, 0.25, 0.5, 0.75, 0.9] quantiles and observed streamflow values plotted chronologically. In this case the post-processed predictions do not show any obvious trend with time, and the widths of the quantile intervals seem to cover the expected number of observed values.

\section{Results}

In this section, we assess the quality of the probabilistic predictions generated by using the three methods and evaluate 


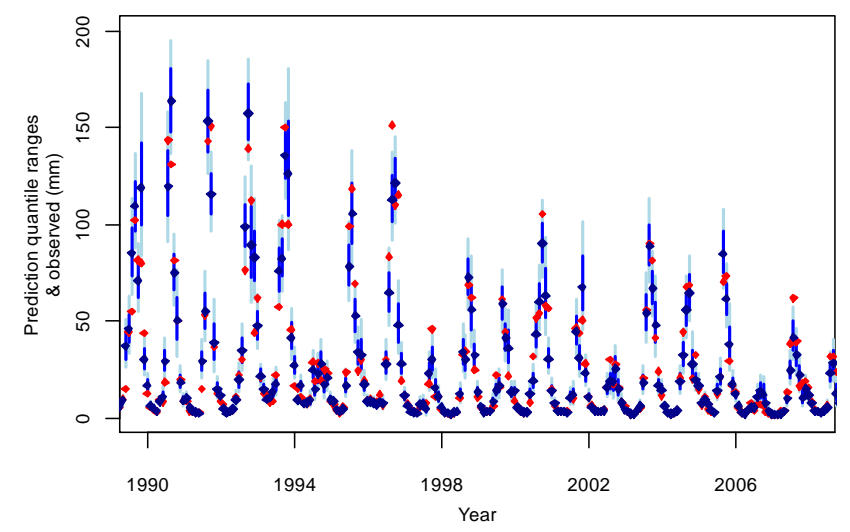

Fig. 2. The time series of the post-processed prediction quantiles against the observed; only a subset of the entire cross validation period is shown (Light blue lines represent 0.1-0.9 quantiles, dark blue lines represent the 0.25-0.75 quantiles, and blue dots represent the medians of the post-processed predictive distributions. Red dots are the observed streamflow values.).

how effective the BJP post-processor is in reducing errors and quantifying uncertainty.

\subsection{Reduction of error}

We assess the ability of the BJP post-processor to reduce errors by using a measure of accuracy called root mean squared error in probability (RMSEP; Wang and Robertson, 2011). RMSEP (Eq. 2) measures error in a probability space. An advantage of RMSEP over the more commonly used mean squared error or root mean squared error is that it places equal emphasis on errors obtained at all events rather than on a few large errors occurring at large events.

$\mathrm{RMSEP}=\left[\frac{1}{n} \sum_{t=1}^{n}\left(F_{\mathrm{CLI}}\left(y^{t}\right)-F_{\mathrm{CLI}}\left(y_{\mathrm{OBS}}^{t}\right)\right)^{2}\right]^{\frac{1}{2}}$

where $y^{t}$ and $y_{\mathrm{OBS}}^{t}$ are the predictions and observations at $t=1,2 \ldots n$ events respectively. The predictions can be either WAPABA simulations or the medians of post-processed distributions. $F_{\mathrm{CLI}}$ is the cumulative historical distribution, and $F_{\mathrm{CLI}}(y)$ is the non-exceedance probability.

\subsubsection{Performance of the WAPABA model}

The RMSEP error values of the WAPABA predictions are shown in Fig. 3. Each row in the figure corresponds to a catchment and each column to a month. In general, except for Cape River and in Queensland, Thompson and O'Shannassy reservoirs in southern Victoria, the RMSEP values are relatively higher in drier months or months when the catchments just start to get wet. This occurs during August-October in Queensland, May-March in upper Murray, January-May in central Victoria and February-March in Tasmania.

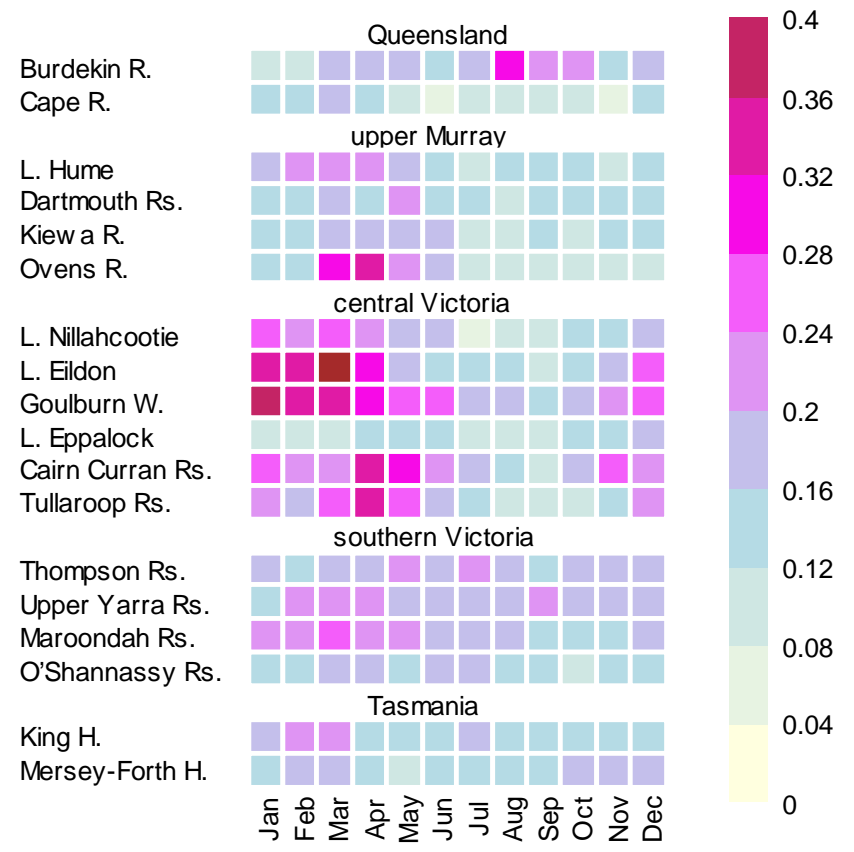

Fig. 3. Performance of WAPABA in all 18 catchments in terms of RMSEP values calculated for each month.

This suggests an inability of the model to properly characterize low flows and to capture the change in catchment dynamics from being dry to getting wet. There can be various reasons for this, including, for example, non-consistency in the data between the calibration and evaluation period (with the calibration period being either wetter or drier), the choice of objective function for calibration, inadequate model structure or a combination of these. While it might be interesting to investigate the causes of poor model performance in these catchments from a model diagnostic point of view, this is beyond the scope of this study. Here we only focus on evaluating whether the errors can be reduced by the post-processor.

\subsubsection{Method A: bias reduction}

Figure 4a shows the differences in RMSEP error values between the WAPABA predictions and those produced from method A (WAPABA prediction - method A). The values are colour coded with blue indicating the reductions in RMSEP error values and red indicating increases.

In general, the result shows that method A effectively reduces systematic bias present in WAPABA predictions. This is manifested as reductions in RMSEP error values over the 18 catchments. The reductions in RMSEP roughly follow the error patterns seen in Fig. 3. In most cases, the differences in RMSEP values are either positive or zero, indicating that the post-processor either reduces errors or preserves (does not degrade) performance of the WAPABA predictions. The highest reductions in RMSEP values occur in Lake Eildon and Goulburn Weir of central Victoria. 
(a)

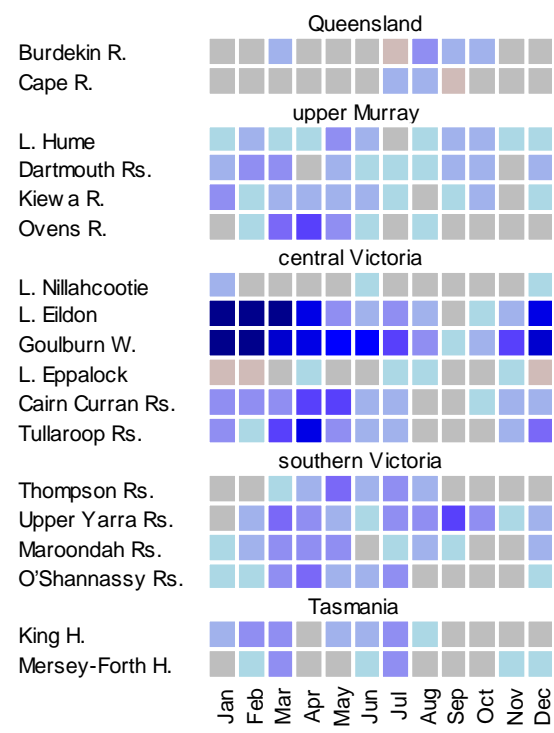

(b)

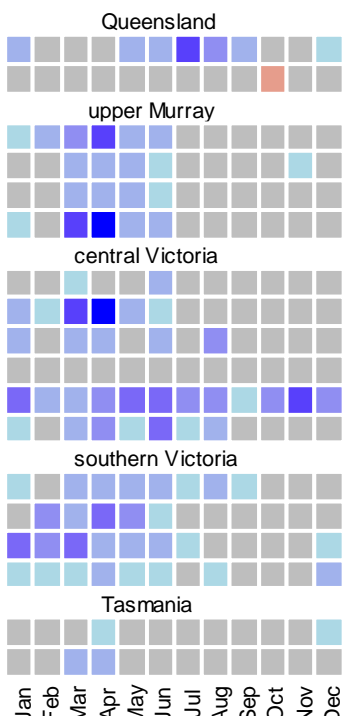

(c)

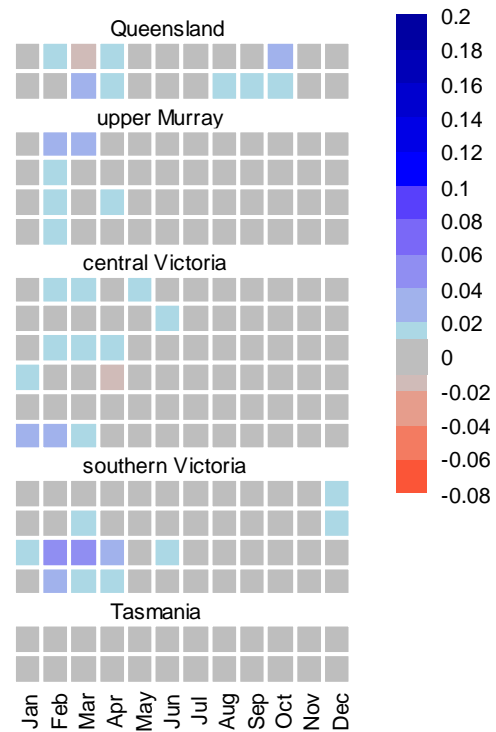

Fig. 4. (a) Difference in RMSEP values between WAPABA predictions and method A (WAPABA - method A); (b) difference in RMSEP values between method A and B (method A -method B); (c) difference in RMSEP values between method B and C (method B - method C).

\subsubsection{Method B: prediction updating}

Figure $4 \mathrm{~b}$ shows the benefit of prediction updating by assimilating the recent streamflow observations (method $\mathrm{B})$. We use the difference between methods $\mathrm{A}$ and $\mathrm{B}$ (method A - method B) to indicate any further reductions in errors achieved by prediction updating. As in the previous case, blue indicates reductions in errors and red indicates increases.

The figure shows further reductions in RMSEP values after bias correction (method A). The reductions occur in most of the catchments. The reductions in errors are governed by whether the errors are present after bias correction and the persistence in the streamflow observation data. For example, the WAPABA predictions in Cape River of Queensland and Lake Nillahcootie of central Victoria (Fig. 3) show the presence of substantially large error values in the initial few months even after bias correction, but they cannot be corrected due to the lack of persistence in the errors. In the upper Murray region, central Victoria and southern Victoria, reductions occur in most of the catchments, and in some catchments (such as Cairn Curran Reservoir) it is greater than that achieved through bias correction. In Tasmanian catchments, the reductions are negligible.

\subsubsection{Method C: prediction updating using WAPABA lagged simulation}

Figure $4 \mathrm{c}$ shows additional benefits achieved by assimilating "lagged" streamflow simulation. The difference is measured relative to method $\mathrm{B}(\operatorname{method} \mathrm{B}-\operatorname{method} \mathrm{C})$ such that posi- tive (blue) values indicate further reductions in RMSEP error values over that achieved by method $B$. The result shows that the benefits of adding lag-1 WAPABA streamflow tend to be negligible in most catchments and seasons. However, some reductions in RMSEP error values can be observed in Maroondah reservoir (in southern Victoria), for the months of February, March and May. In other catchments, the differences in RMSEP values are close to zero. This suggests that two predictors in the BJP post-processors (WAPABA prediction and lag-1 streamflow observation) are able to capture all information about the residual error structure from the training data, thus making contributions from an additional predictor redundant.

\subsection{Quantification of uncertainty}

The post-processor should be able to quantify the uncertainty in predictions. As a measure of the ability to quantify uncertainty, we assess if the probabilistic predictions generated by the post-processor are reliable and robust. We assess the predictions generated using all three methods in 18 catchments but present results for Lake Eildon using method B as a general representation.

\subsubsection{Assessment of reliability}

Reliability refers to "statistical consistency" of the predictive probability distributions with the observed frequency of the events (Toth et al., 2003; Robertson et al., 2012). In this study, we use PIT (probability integral transform) uniform probability plots (Wang et al., 2009; Wang and Robertson, 2011) to assess the overall reliability of the post-processed 


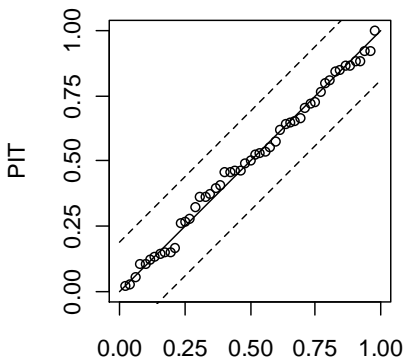

Standard Uniform variate

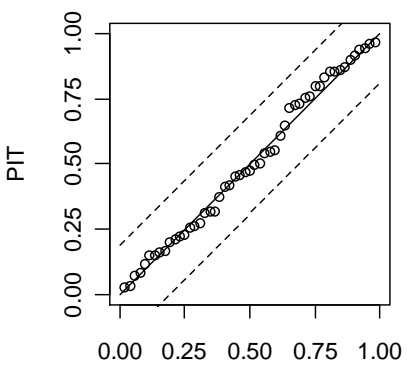

Standard Uniform variate
Fig. 5. PIT uniform probability plots of post-processed streamflow predictions for two months (February and July) in Lake Eildon (1: 1 solid line, theoretical uniform distribution; broken lines, Kolmogorov $5 \%$ significance band; circle, PIT value of observed streamflow).

predictive distributions. We choose PIT uniform probability plots over other methods because they are more suited to smaller sample sizes (Wang et al., 2009).

The PIT of the observed value is given as $\pi^{t}=F^{t}\left(y_{\mathrm{OBS}}^{t}\right)$, where $F^{t}\left(y_{\mathrm{OBS}}^{t}\right)$ is the non-exceedance probability of the observed streamflow in the predictive distribution. The predictive distributions are said to be reliable if the PIT values are distributed uniformly. To check uniformity, we plot PIT values corresponding to each event in a uniform probability plot (Wang et al., 2009; Wang and Robertson, 2011). A close alignment of the values to $1: 1$ indicates uniformity and therefore reliable distributions. Deviations from the $1: 1$ line indicate if the predictive distributions are too low or high and if the uncertainty spreads are too wide or narrow. The details on how to interpret the PIT plots can be found in Thyer et al. (2009), Wang et al. (2009) and Wang and Robertson (2011).

Figure 5 shows the PIT uniform probability plots of the post-processed predictions generated for the months of February and July in Lake Eildon. The dotted inclined lines depict the Kolmogorov $5 \%$ significance band. The PIT values in the plots align quite uniformly along the diagonal $1: 1$ line (solid inclined line) and are well within the significance band. This suggests that the post-processed predictive distributions are overall reliable and the width of uncertainty intervals are of appropriate spread (not too wide or narrow). The result is similar for all the months in Lake Eildon (figures not included).

\subsubsection{Assessment of robustness}

Robustness refers here to "conditional reliability" of the predictive distributions over time and event size. To measure the robustness of the predictive distributions against time, we plot PIT values chronologically and analyse the plot for the presence of any trends or patterns. The distributions are robust (over time) if the PIT values are distributed uniformly. Any existing trends or patterns indicate the presence of sys-
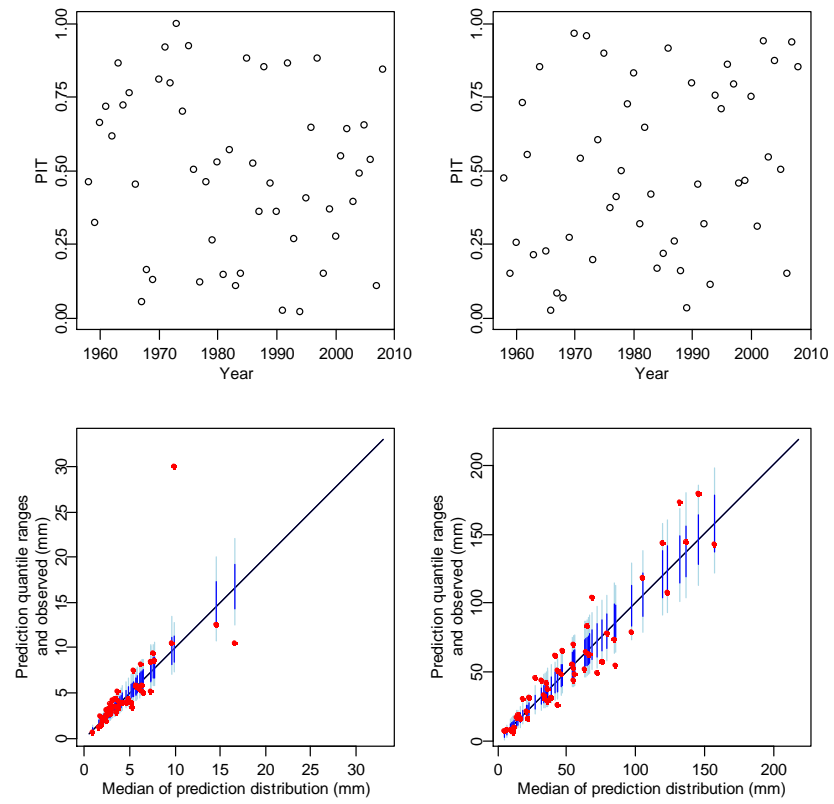

Fig. 6. Top row - probability integral transform (PIT) values plotted against time for the months of February and July; bottom row post-processed quantiles plotted against their median values for the months of February and July. The red dots represent the observed streamflows, light blue vertical lines represent the 0.1-0.9 quantiles and the dark blue lines represent the $0.25-0.75$ quantiles. The $1: 1$ lines represent the forecast median.

tematic errors in the distributions (Wang et al., 2009; Wang and Robertson, 2011).

Figure 6 (top row) shows the PIT values plotted chronologically for February and July. The PIT values tend to be distributed randomly against time, devoid of any trends or patterns indicating that distributions are robust. In fact, this was the case for all the months in Lake Eildon (figure not included).

To measure robustness of the post-processed predictions against flow magnitudes, we plot post-processed prediction quantiles and the observed streamflow values against the medians of the predictions. As in the previous case, we analyse the plot to detect presence of any trends or patterns. Figure 6 (bottom row) shows the post-processed quantiles plotted against event magnitude.

The figure shows that the quantiles increase with event sizes and the medians are consistent with the observed flows. The observed flows are scattered randomly about the medians, suggesting that the post-processed quantiles are robust with respect to event magnitudes. The plots also show that the width of the uncertainty intervals are of appropriate spread for all the event size.

This verification approach is applied for all postprocessing method (A, B and C) for all the catchments, for each month. In general, the results are consistent to the results obtained in Lake Eildon. 


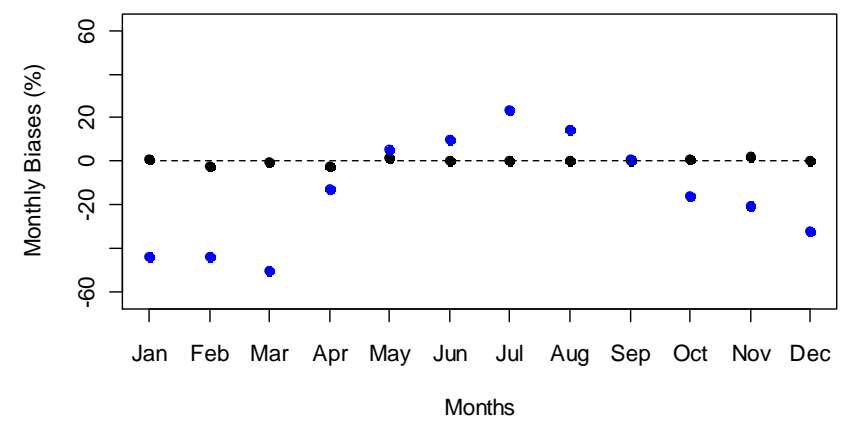

Fig. 7. Monthly biases (\%) in Lake EILDON, raw WAPABA predictions (blue) and method A (black) [The bias for method A = (mean monthly streamflow observations - mean probabilistic predictions averaged each month $) /($ Average observed monthly streamflow $)$; the bias for WAPABA $=$ (mean monthly streamflow observations mean monthly WAPABA predictions)/(Average observed monthly streamflow)].

\section{Discussion}

The results show that large bias can occur in predictions despite calibrating WAPABA using a multicriteria objective function that includes a symmetric measure of bias. This is not surprising because maximization of the scalarized function is a result of compromise between four objective functions and does not necessarily lead to removal of systematic bias in all catchments and in all months. The presence of bias is especially high in Lake Eildon. The BJP post-processor eliminates bias in the predictions effectively, resulting in bias close to zero throughout the year. This can be better appreciated in Fig. 7, which shows monthly percentage bias obtained by WAPABA predictions and its elimination by method A.

Furthermore, it is interesting to note that the bias correction is not just due to linear changes in slope or intercepts but also due to non-linear changes as illustrated by Fig. 8 . The figures demonstrate non-linear compensations to WAPABA predictions by the BJP post-processor. The log-sinh transformation in combination with the BJP model parameter inference allow for the non-linear corrections of errors, thus allowing for corrections of conditional as well as unconditional biases.

Our results show that further error reductions can be possible through prediction updating. This contradicts the assumptions made by Li et al. (2011), who assume that persistence in error structure at monthly time step is negligible. However, we note that the results tend to be catchment specific. In our case the improvements are mostly seen in catchments that have substantial streamflow contribution from the slow responding mechanisms (resulting in longer memory) in the catchment. This seems to be the case in upper Murray, central Victoria and southern catchments, where significant reductions in errors can be observed. The two catchments in Tasmania, the one in Queensland and the one in central Victoria have shorter catchment "memory" with the streamflow
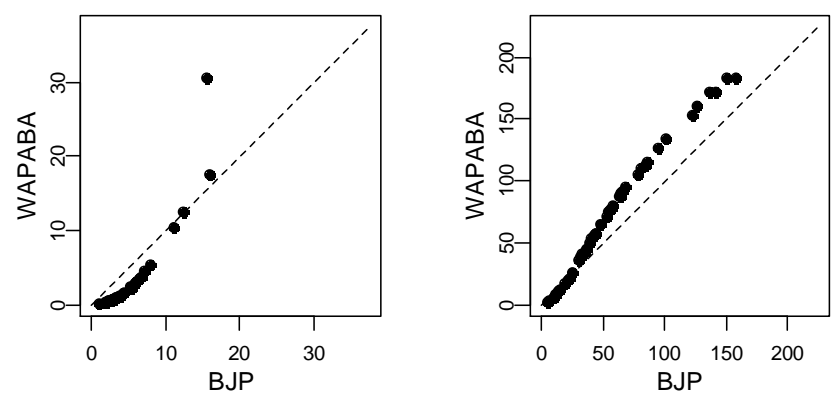

Fig. 8. WAPABA predictions vs. the medians of post-processed predictions produced by method A in Lake Eildon for the months of February (left) and July (right), showing the example of non-linear error corrections by the BJP post-processor.
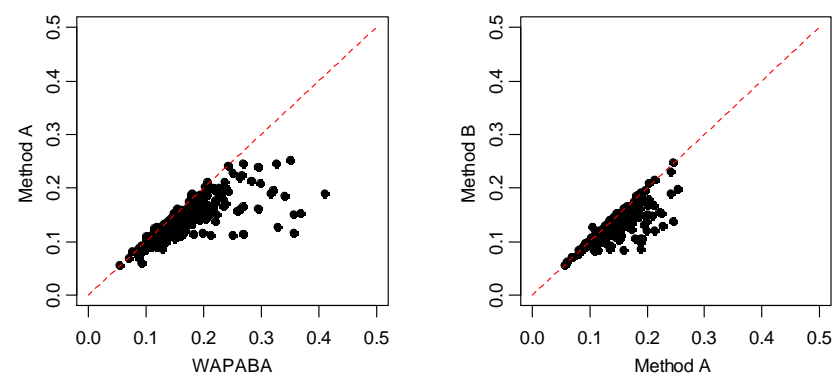

Fig. 9. Scatter plots of RMSEP values; (left) WAPABA predictions vs. method A, (right) method A vs. method B.

being dominated by fast-responding runoff processes, and therefore the benefits of prediction updating are negligible.

In general, the reduction of errors by the post-processor does not necessarily depend upon the magnitude of errors and occurs for small as well as large errors (Fig. 9). However, reductions of errors are not possible in all situations, as illustrated by the points lying in the $1: 1$ lines. As with all the statistical methods, the effectiveness of the BJP postprocessor depends upon the correlation between predictand and predictors, stationarity in relationship (between predictors and predictands) and persistence in the error structure that allow for prediction updating. The post-processor is not effective in situations where none of these occur; this seem to be the case for many points lying in the $1: 1$ lines, most prominent among them being the high RMSEP error values $(>0.25)$ corresponding to predictions in Nillahcootie (see Figs. 9 and 3). However, more importantly the BJP postprocessor is able to preserve skill (not degrade performance) of WAPABA prediction even when error correction is not possible.

We acknowledge that the rainfall forecast uncertainty represents a major source of uncertainty in streamflow forecast (Krzysztofowicz, 1999; Kuczera et al., 2006). In this study, however, we run the water balance model in a simulation mode. Therefore the total uncertainty quantified by the postprocessor is the "lumped" combination of the hydrologic 
uncertainty, rainfall measurement uncertainty, the streamflow measurement uncertainty and the uncertainty in inferring the values of the parameters of the BJP post-processor.

However, the post-processor is equally applicable in real world applications using rainfall forecast ensembles. In such cases the hydrologic model could be forced with rainfall forecast ensembles to create streamflow forecast ensembles. The streamflow forecast ensembles could then be postprocessed to reduce errors and further quantify hydrologic uncertainty in the streamflow forecast (Seo et al., 2006). An alternative approach would be to force the hydrologic model using the mean of rainfall forecast ensembles, then train the post-processor on the deterministic streamflow forecast produced by the hydrologic model, and finally post-process the deterministic forecast to reduce error and quantify the total uncertainty (Pokhrel et al., 2012).

\section{Summary and conclusions}

In this study, we present a statistical post-processor capable of reducing errors and quantifying uncertainty in monthly streamflow predictions. The statistical post-processor is based on the BJP modelling approach (Wang et al., 2009). The BJP post-processor is applied to 18 catchments in Australia, and its ability to reduce errors, through reductions of systematic bias and prediction updating, and to quantify uncertainty in the monthly streamflow predictions is assessed.

The study shows that the BJP post-processor is capable of improving the accuracy of the streamflow predictions by reducing systematic bias in most of the catchments. In many cases, reduction of bias is achieved by means of a nonlinear relationship between model predictions and the observed streamflow values. The post-processor also demonstrates its useful property in preserving the accuracy (does not increase error) of predictions when bias correction is not possible.

Prediction updating through the assimilation of recent streamflows by the post-processor results in further reductions in RMSEP error values over those achieved by bias correction alone, and it is most effective for catchments showing stronger persistence in the prediction errors. Benefits of prediction updating using additional information from the water balance model simulation at the previous time step seem to be very marginal and do not justify the added complexity of introducing another predictor to the post-processor.

The BJP post-processor is capable of generating probabilistic predictions that are overall reliable. The uncertainty quantified by the processor is of appropriate spread. The post-processed predictive distributions are robust with respect to time and event magnitude.

Acknowledgements. This research has been supported by the Water Information Research and Development Alliance between the Australian Bureau of Meteorology and CSIRO Water for a Healthy
Country Flagship, the South Eastern Australian Climate Initiative, and the CSIRO OCE Science Leadership Scheme. Streamflow and GIS data were provided by the Murray-Darling Basin Authority, Melbourne Water, HydroTasmania, Goulburn-Murray Water, the Australian Bureau of Meteorology and the Queensland Department of Environment and Resource Management. We would like to acknowledge James C. Bennett and Roger Hughes for their useful comments and suggestions as well as their help in editing the manuscript. We would also like to thank the two anonymous reviewers for their insightful comments and suggestions, which have helped to improve the paper substantially.

Edited by: M. Werner

\section{References}

Duan, Q., Sorooshian S., and Gupta V. K.: Optimal use of the SCEUA global optimization method for calibrating watershed models, J. Hydrol., 158, 265-284, 1994.

Engeland, K., Xu, C. Y., and Gottschalk, L.: Assessing uncertainties in a conceptual water balance model using Bayesian methodology, Hydrol. Sci. J., 50, 45-63, 2005.

Gupta, H. V., Beven, K. J., and Wagener, T.: Model calibration and uncertainty estimation, in: Encyclopedia of Hydrological Sciences, edited by: Anderson, M., John Wiley \& Sons Ltd: Chichester, 1-17, 2005.

Hashino, T., Bradley, A. A., and Schwartz, S. S.: Evaluation of biascorrection methods for ensemble streamflow volume forecasts, Hydrol. Earth Syst. Sci., 11, 939-950, doi:10.5194/hess-11-9392007, 2007.

Jones, D. A., Wang, W., and Fawcett, R.: High-quality spatial climate data-sets for Australia, Aust. Meteorol. Ocean. J., 58, 233248, 2009.

Krzysztofowicz, R.: Transformation and normalization of variates with specified distributions, J. Hydrol., 197, 286-292, 1997.

Krzysztofowicz, R.: Bayesian theory of probabilistic forecasting via deterministic hydrologic model, Water Resour. Res., 35, 27392750, 1999.

Krzysztofowicz, R.: Bayesian system for probabilistic river stage forecasting, J. Hydrol., 268, 16-40, 2002.

Kuczera, G., Kavetski, D., Franks, S., and Thyer, M.: Towards a Bayesian total error analysis of conceptual rainfall-runoff models: Characterising model error using storm-dependent parameters, J. Hydrol., 331, 161-177, doi:10.1016/j.jhydrol.2006.05.010, 2006.

Lekkas, D. F., Lees, M. J., and Imrie, C. E.: Improved nonlinear transfer function and neural network methods of flow routing for real-time forecasting, J. Hydroinform., 3, 153-164, 2001.

Li, L., Xia, J., Xu, C.-Y., and Singh, V. P.: Evaluation of the subjective factors of the GLUE method and comparison with the formal Bayesian method in uncertainty assessment of hydrological models, J. Hydrol., 390, 210-221, 2010.

Li, L., Xu, C.-Y., Xia, J., Engeland, K., and Reggiani, P.: Uncertainty estimates by Bayesian method with likelihood of AR (1) plus Normal model and AR (1) plus Multi-Normal model in different time-scales hydrological models, J. Hydrol., 406, 54-65, doi:10.1016/j.jhydrol.2011.05.052, 2011.

Montanari, A. and Grossi, G.: Estimating the uncertainty of hydrologic forecasts: a statistical approach, Water Resour. Res., 44, 
W00B08, doi:10.1029/2008WR006897, 2008.

Morawietz, M., Xu, C.-Y., and Gottschalk, L.: Reliability of autoregressive error models as post-processors for probabilistic streamflow forecasts, Adv. Geosci., 29, 109-118, doi:10.5194/adgeo29-109-2011, 2011.

Nash, J. E. and Sutcliffe, J. V.: River flow forecasting through conceptual models part I - A discussion of principles, J. Hydrol., 10, 282-290, 1970.

Pagano, T. C., Wang, Q. J., Hapuarachchi, P., and Robertson, D.: A dual-pass error-correction technique for forecasting streamflow, J. Hydrol., 405, 367-381, 2011.

Pokhrel, P., Wang, Q. J., and Robertson, D. E.: Combining multiple statistical and dynamical forecast models to improve seasonal streamflow forecasts, Water Resour. Res., in review, 2012.

Reggiani, P. and Weerts, A. H.: A Bayesian approach to decisionmaking under uncertainty: An application to real-time forecasting in the river Rhine, J. Hydrol., 356, 56-69, 2008.

Robertson, D. E., Pokhrel, P., and Wang, Q. J.: Improving statistical forecasts of seasonal streamflows using hydrological model output, Hydrol. Earth Syst. Sci. Discuss., 9, 8701-8736, doi:10.5194/hessd-9-8701-2012, 2012.

Seo, D.-J., Herr, H. D., and Schaake, J. C.: A statistical postprocessor for accounting of hydrologic uncertainty in short-range ensemble streamflow prediction, Hydrol. Earth Syst. Sci. Discuss., 3, 1987-2035, doi:10.5194/hessd-3-1987-2006, 2006.

Shamseldin, A. Y. and O'Connor, K. M.: A non-linear neural network technique for updating of river flow forecasts, Hydrol. Earth Syst. Sci., 5, 577-598, doi:10.5194/hess-5-577-2001, 2001.

Shi, X., Wood, A. W., and Lettenmaier, D. P.: How Essential is Hydrologic ModelCalibration to Seasonal Streamflow Forecasting?, J. Hydrometeorol., 9, 1350-1363, 2008.

Thyer, M., Renard, B., Kavetski, D., Kuczera, G., Franks, S. W., and Srikanthan, S.: Critical evaluation of parameter consistency and predictive uncertainty in hydrological modeling: A case study using Bayesian total error analysis, Water Resour. Res., 45, W00B14, doi:10.1029/2008WR006825, 2009.
Todini, E.: A model conditional processor to assess predictive uncertainty in flood forecasting, Int. J. River Basin Manage., 6, 123-137, 2008.

Toth, Z., Talagrand, O., Candille, G., and Zhu, Y.: Probability and Ensemble Forecasts, in: Forecast Verification: A Practitioner's Guide in Atmospheric Science, edited by: Jolliffe, I. T. and Stephenson, D. B., J. Wiley, Chichester, 137-164, 2003.

Wang, Q. J. and Robertson, D. E.: Multisite probabilistic forecasting of seasonal flows for streams with zero value occurrences, Water Resour. Res., 47, W02546, doi:10.1029/2010wr009333, 2011.

Wang, Q. J., Robertson, D. E., and Chiew, F. H. S.: A Bayesian joint probability modeling approach for seasonal forecasting of streamflows at multiple sites, Water Resour. Res., 45, W05407, doi:10.1029/2008WR007355, 2009.

Wang, Q. J., Pagano, T. C., Zhou, S. L., Hapuarachchi, H. A. P., Zhang, L., and Robertson, D. E.: Monthly versus daily water balance models in simulating monthly runoff, J. Hydrol., 404, 166-175, 2011.

Wang, Q. J., Shrestha, D. L., Robertson, D. E., and Pokhrel, P.: A log-sinh transformation for data normalization and variance stabilization, Water Resour. Res., 48, W05514, doi:10.1029/2011WR010973, 2012.

WMO: Simulated real-time intercomparison of hydrological models, Op. Hydrol. Rep. World Meteorological Organisation, Geneva, 1992.

Xiong, L. H. and O'Connor, K. M.: Comparison of four updating models for real-time river flow forecasting, Hydrol. Sci. J., 47, 621-639, 2002.

Yeo, I. and Johnson, R. A.: A new family of power transformations to improve normality or symmetry, Biometrika, 87, 954959, 2000.

Zhang, L., Potter, N., Hickel, K., Zhang, Y., and Shao, Q.: Water balance modelling over variable time scales based on the Budyko framework - model development and testing, J. Hydrol., 360, 117-131, 2008.

Zhao, L., Duan, Q., Schaake, J., Ye, A., and Xia, J.: A hydrologic post-processor for ensemble streamflow predictions, Adv. Geosci., 29, 51-59, doi:10.5194/adgeo-29-51-2011, 2011. 